\title{
Satiety effects of a physiological dose of cholecystokinin in humans
}

\author{
R J Lieverse, J B M J Jansen, A A M Masclee, C B H W Lamers
}

\begin{abstract}
Cholecystokinin 33 (CCK) was infused intravenously to eight healthy obese women and 10 healthy lean women of the same age, in doses that elicited plasma cholecystokinin concentrations in the physiological range. The effect of these infusions after a standardised banana 'shake' (preload) on food intake and satiety signals was compared with the effect of saline infusions in the same subjects. For the whole group food intake (mean (SEM)) (282 (29 g)) was significantly less during CCK than during saline (346 (31) g, p<0.05). Hunger feelings tended to be less during CCK infusions. Examination of the separate subgroups showed no differences between lean and obese subjects in the satiety effects of CCK. In conclusion, under the conditions of this study, CCK significantly decreases food intake in humans, and this effect is similar for lean and obese subjects.

(Gut 1995; 36: 176-179)
\end{abstract}

Keywords: cholecystokinin, satiety, obesity.

Many studies have shown that cholecystokinin (CCK) reduces food intake in several species including humans. ${ }^{1-14}$ In these studies, however, CCK plasma concentrations were not measured and the reported feeding depression may well have been caused by supraphysiological doses of CCK. Wolkowitz on the other hand reported that CCK receptor blockade increased hunger in humans showing that CCK may have a physiological satiety effect also in humans. ${ }^{15}$ It has recently been shown that an infusion of CCK 33 leading to high physiological plasma concentrations does not have an important effect on food intake and postprandial satiation in humans in a study where no preload was given. ${ }^{16} \mathrm{~A}$ gastric load, however, potentiates the inhibition of food intake produced by cholecystokinin and reduces the threshold dose of a CCK analogue required to inhibit food intake in monkeys and rats. ${ }^{12} 17$ In the human studies of Pi-Suyner and Kissileff where a CCK 8 infusion diminished food intake in lean and obese humans, an appetiser, consisting of crackers and jelly $(216 \mathrm{kcal})$ was given 12 minutes before the start of the liquid meal. ${ }^{34}$ Muurahainen et al reported that a $500 \mathrm{~g}$ but not $100 \mathrm{~g}$ soup preload combined with CCK $8225 \mathrm{ng} / \mathrm{ml} / \mathrm{min}$ significantly decreased food intake in nonobese humans compared with the same preload with a saline infusion. ${ }^{18}$ We therefore studied the effects of intravenous infusion of
CCK 33, in a dose that leads to high physiological plasma concentrations, on food intake, 15 minutes after a preload, and satiety signals in lean and obese volunteers. Apart from the preload this study design is comparable with our previous investigation. ${ }^{16} \mathrm{We}$ infused $\mathrm{CCK}$ 33 into the volunteers, as we have shown that CCK 33 like immunoreactivity represents one of the important circulating molecular forms in humans, whereas CCK 8 was almost absent. ${ }^{19}$ Given that the octapeptide of CCK is almost absent in human plasma it is probable that the human studies on CCK 8 and satiety examined a pharmacological effect rather than the physiological effect of CCK that we investigated using CCK 33.

\section{Methods}

Eight healthy obese women (age 41 (3) years, body mass index of $39(2) \mathrm{kg} / \mathrm{m}^{2}$ ) and 10 age and sex matched healthy lean women (age 41 (2) years with a body mass index of 22 (3) $\mathrm{kg} / \mathrm{m}^{2}$ ) were studied. Informed consent was obtained from all subjects. The investigations were approved by the local human ethics committee.

After an overnight fast the volunteers came to the laboratory at 0800 . Saline or highly purified CCK 33 (1 IDU/kg ideal weight/ height, Karolinska Institute, Stockholm, Sweden) was infused through an intravenous catheter for 165 minutes, in random order and double blinded. The subjects actual weight was used as the ideal weight in the lean subjects and for obese subjects their height in $\mathrm{cm}$ was subtracted by 100 because it was known from previous experiments that this resulted in comparable plasma concentrations. ${ }^{16}$ The infusion was started at 0900 . The two studies were separated from each other by at least one week. The subjects were investigated on days irrespective of their time in the menstrual cycle. Sixty minutes after the start of the saline or CCK infusion, a banana 'shake' consisting of $100 \mathrm{~g}$ of banana slices, supplemented with $300 \mathrm{ml}$ of water and mixed $(132 \mathrm{kcal})$, was served and consumed within three minutes. Fifteen minutes later, at $t=75$ minutes a solid meal of slices of bananas in abundancy, containing $1 \mathrm{~g} / 100 \mathrm{~g}$ of protein, $0 \mathrm{~g} / 100 \mathrm{~g}$ of fat, and $32 \mathrm{~g} / 100 \mathrm{~g}$ of carbohydrate was served and the meal was weighed before and afterwards to determine the exact amount of food consumed.

Banana slices were chosen because most people like them and they have only minimal CCK stimulating potency as was shown in our previous study without a preload. ${ }^{16}$

Otherwise, if the preload or meal had 

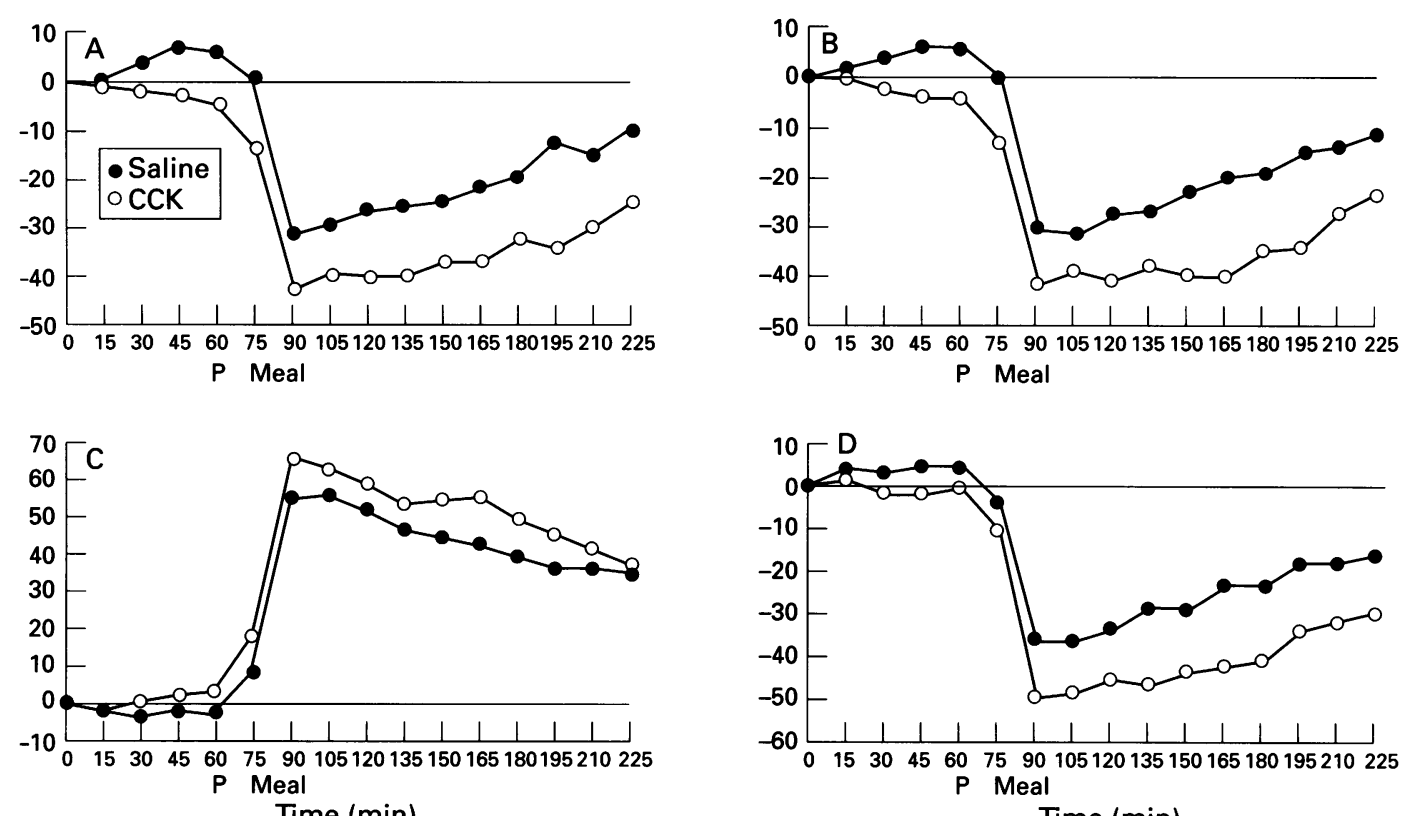

Figure 1: $(A)$ Desire to eat, $(B)$ hunger, $(C)$ fullness, and (D) prospective feeding intentions during saline or cholecystokinin infusions from $t=0$ until $t=165$ minutes in 18 healthy women. After 60 minutes a banana 'shake' (preload $=P$ ) was consumed and 15 minutes later a meal.

stimulated endogenous CCK release this might have led to supraphysiological plasma CCK concentrations in combination with the $\mathrm{CCK}$ infusion. Subjective criteria, like desire
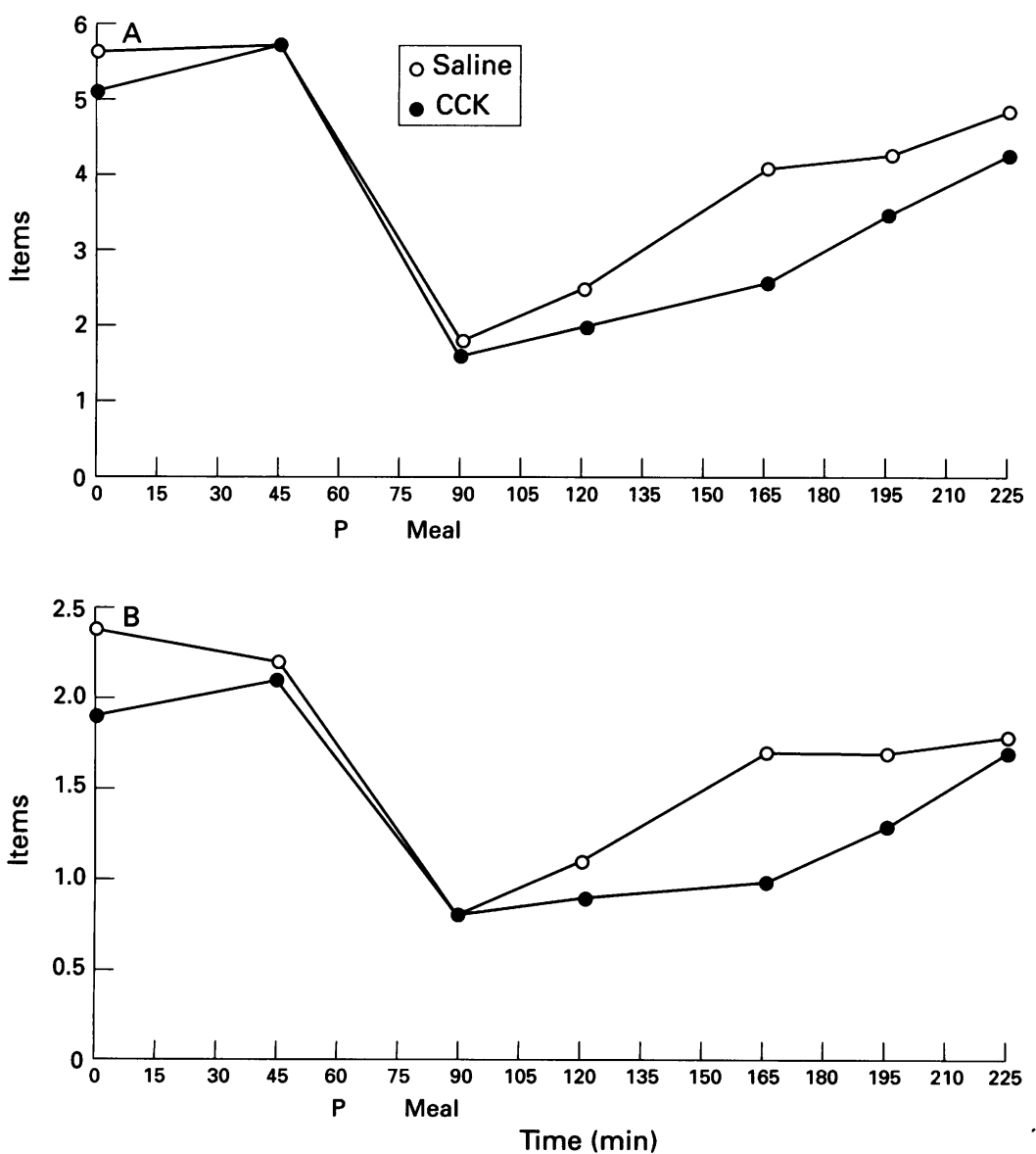

Figure 2: $(A)$ Desire to eat caloric items in 18 healthy women during saline or $C C K$ infusion. After 60 minutes a banana 'shake' (preload $=P$ ) was consumed and 15 minutes later a meal. After the meal CCK induced a significant satiety effect when the incremental area under the curve was analysed $(p<0.05)$. (B) Desire to eat fatty items in 18 healthy women during saline or CCK infusion. After 60 minutes a banana 'shake' (preload $=P$ ) was consumed and 15 minutes later a meal. After the meal CCK induced a significant satiety effect for fatty items when the incremental area under the curve was analysed $(p<0 \cdot 05)$. to eat, hunger feeling, fullness, and prospective feeding intentions were scored on $100 \mathrm{~mm}$ visual analogue scales basally and at 15 minute intervals after the meal until 60 minutes after the end of the infusion period. ${ }^{2021}$ Questions asked were: how strong is your desire to eat (very weak - very strong); how hungry do you feel (not hungry at all - as hungry as I have ever felt); how full do you feel (not at all full - very full); how much food do you think you could eat (nothing - very much). Hunger feelings were also measured with food selection lists as described by Hill ${ }^{22}$ and modified to Dutch feeding customs. Fifteen minutes before the infusion, 15 minutes before the banana 'shake' (at $\mathrm{t}=45$ ), 15 minutes after the meal (at $\mathrm{t}=90$ ), and at $\mathrm{t}=120, \mathrm{t}=165$ (end of the infusion), and $t=225$ (end of the experiment), lists and photographs showing six protein rich, six fat rich, six carbohydrate rich (each (200) kcal), and six low energy items were presented. From each of these 24 items the subjects were asked if they wanted to eat immediately the amount shown, double the amount, half the amount or nothing at all independent of the other items. At each time interval the total amount of caloric items was calculated (half the amount $=1 / 2$ caloric item, double the amount $=2$ caloric items). The subjects also indicated on visual analogue scales if they appreciated the meal and if they experienced nausea. Plasma CCK concentrations were measured by a sensitive and specific radioimmunoassay using antibody $T_{204}$. This antibody binds to all carboxy terminal CCK peptides containing the sulphated tyrosyl region and recognises biologically active CCK forms equally. The detection limit of the assay was $0.5 \mathrm{pmol} / \mathrm{l}$ plasma. All samples were measured in one run. The intra-assay variation ranged from 4.6 to $11.5 \% .{ }^{23} 24$

Results are given as mean (SEM). Statistical analysis of hunger feelings was done by calculating the integrated area under the curve 

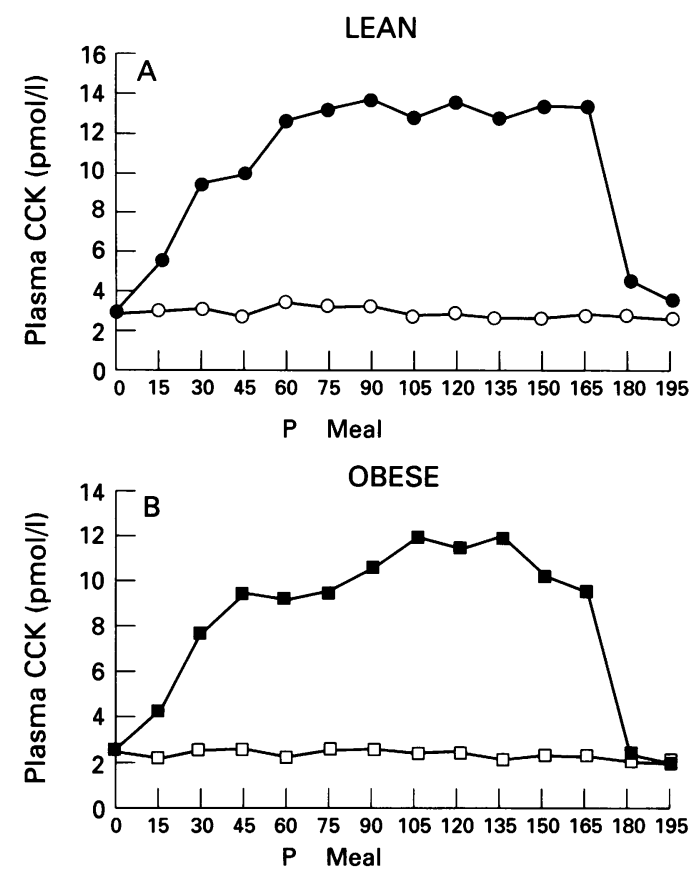

Time (min)

Figure 3: Plasma CCK concentrations during saline (open symbols) or CCK infusion (closed symbols) in $(A)$ lean or (B) obese women. After 60 minutes a banana 'shake' (preload $=P$ ) was consumed and 15 minutes later a meal. Basal concentrations were comparable in lean and obese women as were the plasma concentrations during CCK infusion.

(AUC) before and after the meal followed by Wilcoxon matched pairs, signed rank test. Food intake and plasma CCK concentrations were compared using Wilcoxon's matched pairs signed rank test. Differences between lean and obese subjects were analysed using the Mann-Whitney U test.

\section{Results}

During CCK infusion food intake (282 (29) g) was significantly less for the 18 women than during saline infusion (346 (31) $\mathrm{g}, \mathrm{p}<0.05)$. Subjective criteria, like desire to eat, hunger feelings, and prospective feeding intentions tended to be less during CCK infusions (Fig 1), but the differences did not reach statistical significance $(p<0.06$ for the AUC before and $p=0.09$ for the AUC after the meal for desire to eat and $p=0.08$ for the AUC after the meal for hunger feelings).

There was also a significant satiating effect of CCK after the meal according to the food selection lists $\mathrm{p}<0.05$ (Fig 2). Analysing the protein, fatty, and carbohydrate items separately, this satiating effect of CCK was specific for the fatty items, but not for the protein and carbohydrate rich items.

During CCK infusion in the lean women food intake (295 (45)) was also less than during saline ( $358(43) \mathrm{g}$ ) but this difference just failed to reach significance $(p=0 \cdot 06)$. Also in the obese women CCK infusion induced a feeding depression from 331 (49) $\mathrm{g}$ during saline to 265 (36) $\mathrm{g}$ during CCK infusion (NS). There were also no differences found between lean and obese women with regard to subjective hunger feelings apart from a significantly lower postprandial desire for fatty items in lean women during $\operatorname{CCK}(\mathrm{p}<0.05)$ whereas there was no difference in obese women between saline and CCK.

Basal plasma CCK concentrations were not significantly different before the saline $(2 \cdot 7$ $(0 \cdot 2) \mathrm{pM})$ and CCK infusion $(2.6(0 \cdot 1) \mathrm{pM})$ and not significantly different in lean $(2.9$ $(0.3) \mathrm{pM})$ and obese subjects $(2.5(0.2) \mathrm{pM})$.

Infusion of saline failed to significantly affect basal CCK concentrations in both lean and obese volunteers (Fig 3). Infusion of CCK resulted in significant increases of plasma CCK concentrations to values fluctuating around $12 \mathrm{pM}$ in both lean and obese subjects (Fig 3). To stop the CCK infusion resulted in rapidly declining plasma CCK concentrations, which reached basal values within 30 minutes.

One of the lean subjects experienced a headache during both the saline and CCK experiment and another lean subject developed diarrhoea during the CCK experiment. There were no other adverse effects. In the lean subjects the duration of the meal was significantly shorter with CCK $(5 \cdot 3(0.88) \mathrm{min})$ than with saline $(7.8(1.43) \mathrm{min}, \mathrm{p}<0.05)$ whereas in obese subjects the duration of the meal with CCK (7.25 (1) min) and saline (7.5 (0.71) min) was comparable.

The appreciation of the meal was comparable between the saline and CCK experiment (60 (5) $v 64(6) \mathrm{mm}$ ) and there was no nausea in lean and obese subjects.

\section{Discussion}

This study shows for the first time that exogenously given CCK 33, resulting in plasma CCK concentrations seen after a mixed meal, ${ }^{23}$ significantly diminishes the size of a carbohydrate meal and increases postmeal satiety in humans. There were no clear differences in these satiating effects of CCK between the lean and obese subgroups.

Using the same CCK assay Fried et al found in healthy volunteers a CCK increase from $2 \cdot 6$ $(0.4) \mathrm{pM}$ to a maximum of 13 (4) pM after the ingestion of a mixed meal consisting of $60 \mathrm{~g}$ boiled chicken breast, $40 \mathrm{~g}$ of boiled chicken liver, one slice ( $28 \mathrm{~g})$ of white bread spread with $15 \mathrm{~g}$ of margarine, $50 \mathrm{~g}$ of ice cream, and $250 \mathrm{ml}$ of lemonade $(0.4 \mathrm{M}$ glucose flavoured with lemon concentrate). ${ }^{24}$

This study differs from various other studies in several respects. Firstly, a solid meal contained almost exclusively carbohydrate without recorded CCK stimulation was ingested; secondly, infusion of CCK resulted in physiological plasma CCK concentrations, and thirdly, CCK 33 in contrast with CCK 8 was infused. In the studies on humans by Pi-Sunyer, ${ }^{3}$ Kissileff, ${ }^{4}$ Stacher, ${ }^{10}$ and Shaw ${ }^{11}$ where CCK 8 induced feeding depression, CCK plasma concentrations were not determined and it is probable that supraphysiological, pharmacological plasma concentrations were achieved. Pi-Sunyer and Kissileff infused $4 \mathrm{ng} / \mathrm{kg} / \mathrm{min}$ of CCK 8 , which is equivalent to about $3.6 \mathrm{pmol} / \mathrm{kg} / \mathrm{min}$ of CCK $8 .^{25}$

CCK infusion induced a selective reduction 
of preference for fatty foods. This has also been shown for endogenous $\mathrm{CCK}^{26}$ and it is possible that the suppression of a fatty meal by CCK would be stronger than the suppression of the carbohydrate meal in our experiment.

We are, however, still a long way from using CCK therapeutically as a treatment option for obesity. Unfortunately, CCK has to be given parenterally and has a very short half life in the circulation, making longterm administration difficult. Thus oral or nasal preparations with prolonged action have to be developed or a diet, which powerfully induces endogenous CCK release with a minimum of caloric load, has to be constituted. Another important problem is the lack of studies that show weight reduction during longterm administration. 8913 The meal frequency in rats is increased to compensate for the reduction in meal size ${ }^{57}$ and tolerance for CCK with repeated or continuous infusion supervenes in rats. ${ }^{627}$ It may be possible that CCK has to be combined with other satiation signals to induce weight reduction.

In conclusion, this study has shown that infusion of CCK 33 leading to plasma concentrations comparable with those after a meal decreases food intake in humans and that this effect is not different for lean and obese subjects.

This work was supported by grant 900-522-080 from the Netherlands Organisation for Scientific Research (NWO). The authors are indebted to Mrs D M Mooy for technical support, Mrs M Koster-de Vreese, and Ms J W van Spronsen for secreMrs M Koster-de
tarial assistance.

1 Stacher G. Effects of cholecystokinin and caerulein on human eating behavior and pain sensation: a review. Psychoneuroendocrinology 1986; 11: 39-48.

2 Morley JE. Neuropeptide regulation of appetite and weight. Endocr Rev 1987; 8: 256-87.

3 Pi-Sunyer X, Kissileff HR, Thornton J, Smith GP. C-Terminal octapeptide of cholecystokinin decreases food intake in obese man. Physiol Behav 1982; 29: food intak

4 Kissileff HR, Pi-Sunyer X, Thornton J, Smith GP. C-terminal octapeptide of cholecystokinin decreases food intake in man. Am $\mathcal{F}$ Clin Nutr 1981; 34: 154-60.

5 West DB, Fey D, Woods SC. Cholecystokinin persistently suppresses meal size but not food intake in free-feeding rats. Am $\mathcal{F}$ Physiol 1984; 246: R776-87.

6 Mineka S, Snowdon CT. Inconsistency and possible habituation of CCK-induced satiety. Physiol Behav 1987; 21: $65-72$.
7 McLaughlin CL, Peikin SR, Baile CA. Food intake response to modulation of secretion of cholecystokinin in Zucker rats. Am f Physiol 1983; 244: R676-85.

8 McLaughlin CL, Peikin SR, Baile CA. Trypsin inhibitor effects on food intake and weight gain in Zucker rats. Physiol Behav 1983; 31: 487-91.

9 McLaughlin CL, Baile CA, Buonomo FC. Effect of CCK antibodies on food intake and weight gain in Zucker rats. Physiol Behav 1985; 34: 277-82.

10 Stacher G, Steinringer H, Schmierer G, Schneider C, Winklehners $S$. Cholecystokinin octapeptide decreases intake of solid food in man. Peptides 1982; $1: 133-6$

11 Shaw MJ, Hughes J, Morley JE, Levine AS, Silvis SE, Shafer RB. Cholecystokinin octapeptide action on gastric emptying and food intake in normal and vagotomized man. Ann NY Acad Sci 1985; 448: 640-1.

12 McHugh PR, Moran TH. The stomach, cholecystokinin and satiety. Fed Proc 1986; 45: 1384-90.

13 Smith GP. The therapeutic potential of cholecystokinin. Int $f$ Obes 1984; (suppl 1): 35-8.

14 Lieverse RJ, Jansen JBMJ, Lamers CBHW. Cholecystokinin and satiation. Neth $\mathcal{J}$ Med 1993; 42: 146-52.

15 Wolkowitz OM, Gertz B, Weingartner H, Beccaria L, Thompson K, Liddle RA. Hunger in humans induced by MK-329, a specific peripheral-type cholecystokinin receptor antagonist. Biol Psychiatry 1990; 28: 169-73.

16 Lieverse RJ, Jansen JBMJ, Zwan A vd, Samson L, Masclee AAM, Lamers CBHW. Effects of a physiological dose of cholecystokinin on food intake and postprandial satiation in man. Regul Pept 1993; 43: 83-9.

17 Schwartz GJ, Netterville LA, McHugh PR, Moran TH. Gastric loads potentiate inhibition of food intake produced by a cholecystokinin analogue. Am $\mathcal{f}$ Physiol 1991; 261: R1141-6.

18 Muurahainen NE, Kissileff HR, Lachaussee J, Pi-Sunyer FX. Effect of a soup preload on reduction of food intake FX. Effect of a soup preload on reduction of food intake
by cholecystokinin in humans. Am $\mathcal{F}$ Physiol 1991; 260: by cholecyst

19 Jansen JBMJ, Lamers CBHW. Molecular forms of cholecystokinin in plasma from normal and gastrectomized human subjects following a fat meal. Peptides 1987; 8 801-5.

20 Blundell JE, Burley VJ. Satiation, satiety and the action of fibre on food intake. Int f Obes 1987; 11: 9-25.

21 Silverstone B. Measurement of hunger and food intake in man. In: Silverstone T, ed. Drugs and appetite. London: Academic Press, 1982: 81-92.

22 Hill AJ. Investigation of short term influence on hunger, satiety and food consumption [thesis]. Leeds: Leeds Satiety and food

23 Jansen JBMJ, Lamers CBHW. Radioimmunoassay of cholecystokinin in human tissue and plasma. Clin Chim Acto 1983; 131: 305-16.

24 Fried M, Mayer EA, Jansen JBMJ, Lamers CBHW, Taylor IL, Bloom SR, et al. Temporal relationships of cholecystokinin release, pancreatobiliary secretion, and gastric emptying of a mixed meal. Gastroenterology 1988; 95: $1344-50$.

25 Reidelberger RD, Kalogeris TJ, Solomon TE. Plasma CCK levels after food intake and infusion of CCK analogues that inhibit feeding in dogs. Am 7 Physiol 1989; 25: R1148-54.

26 Lieverse RI, Jansen JBMJ, Masclee AAM, Rovati LC, Lamers CBHW. Effect of a low dose of intraduodenal fat on satiety in humans: studies using the type A cholecystokinin receptor antagonist loxiglumide. Gut 1994; 35 501-5.

27 Crawley JN, Beinfeld MC. Rapid development of tolerance to the behavioural actions of cholecystokinin. Nature 1983; 302: 703-6. 